\title{
Daily Profiles of Steroid Hormones and Their Metabolites Related to Food Intake
}

\author{
B. RÁCZ ${ }^{1}$, M. DUŠKOVÁ ${ }^{1}$, K. VONDRA ${ }^{1}$, M. ŠRÁMKOVÁ ${ }^{1}$, M. HILL ${ }^{1}$, L. STÁRKA $^{1}$ \\ ${ }^{1}$ Institute of Endocrinology, Prague, Czech Republic
}

Received May 6, 2015

Accepted May 20, 2015

\begin{abstract}
Summary
The aim of this study was to look for changes in the daily profile of steroid hormones after standardized food intake. Eight young women not taking contraceptives were followed from 5:30 a.m. till 9:30 p.m. before and 1 and $2 \mathrm{~h}$ after eating breakfast, snack, lunch, the second snack and dinner. The differences in steroid levels before and after meals were evaluated. As expected, glucose, C-peptide and ghrelin levels changed postprandially. The steroid hormones cortisol, progesterone, pregnenolone and dehydroepiandrosterone showed a decrease after main meals, whereas testosterone and dihydrotestosterone showed no significant dependence on food intake. Estrogen levels did not exhibit a significant nycthemeral rhythm, but estradiol decreased after main meals. In our study the known nycthemeral rhythm of $\mathrm{LH}, \mathrm{FSH}$, cortisol, progesterone and pregnenolone after food intake were confirmed, but significant changes after meals were also observed in the levels of cortisol, dehydroepiandrosterone, estradiol and SHBG.
\end{abstract}

\section{Key words}

Daily pattern • Food intake $\bullet$ Steroid hormones $\bullet$ Ghrelin $\bullet$ Orexin - SHBG

\section{Corresponding author}

M. Dušková, Institute of Endocrinology, Národní 8, 11694 Prague 1, Czech Republic. E-mail: mduskova@endo.cz

\section{Introduction}

A large amount of information has been accumulated on the time structure of the endocrine system. Circadian rhythms of physiological actions including metabolism and behavior are generated by central and peripheral circadian oscillators, which are tuned up by periodic environmental or physiological stimuli. A master circadian pacemaker in the hypothalamic suprachiasmatic nucleus (SCN) is directly controlled by daily changes of light-dark cycles, and coordinates the timing of other oscillators by direct and indirect neuronal, hormonal and behavioral signals. The daily rhythm of food intake creates stimuli that calibrate most peripheral and central oscillators. Some of these could be involved in the daily rhythm of food anticipatory activity (Patton and Mistlberger 2013).

The highly coordinated output of the hypothalamic biological clock not only governs the daily rhythm in sleep/wake (or feeding/fasting) behavior but also has direct control over many aspects of hormone release. In fact, a significant proportion of our current understanding of the circadian clock has its roots in studies of the intimate connections between the hypothalamic clock and multiple endocrine axes (Kalsbeeg and Fliers 2013).

Until now, most attention was paid to the main hormones such as melatonin (Vaněček 1998, Trivedi and Kumar 2014), cortisol (Dickmeis 2009, Chung et al. 2011, Son et al. 2011), testosterone (Karatsoreos et al. 2007), estradiol (Christian and Moenter 2010), the neurohormones oxytocin and vasopressin (Forsling 2000) and enterohormones or hormones related to food intake (Konturek et al. 2011). Only rare reports have dealt with other steroid hormones. Some data were published on dehydroepiandrosterone (Stárka et al. 2015) and its hydroxymetabolites, no data could we find for allopregnanolone or pregnenolone. However, in many situations, particularly for neuroactive metabolites, these steroids can extensively modulate many brain functions like mood or behavior. Reports on the effects of food intake, which could change the basal course of the daily hormone profile, are scanty. 


\section{Materials and Methods}

\section{Subjects}

Eight women were involved in this study. They were 25-35 years old with a mean of 29.48 \pm 2.99 years. Their BMI was normal, with a mean BMI of $21.3 \pm 1.3$. All subjects were premenopausal, non-smokers, healthy and used no medications or hormonal contraception. All were in the follicular phase (days 1-7 of the menstrual cycle) at the time of study. The volunteers were kept in standardized conditions for five days ( $8 \mathrm{~h}$ of sleeping and periodic eating according to a standardized menu, which did not differ significantly from their normal daily activities and habits).

The Ethical Committee of the Institute of Endocrinology in Prague approved the protocol of the study, and written informed consent was obtained from all participants.

During the trial, all of the volunteers consumed similar food with a standard amount of carbohydrates, proteins and lipids, with a total caloric value of about $8400 \mathrm{~kJ}$. The participants ate only meals according to the prepared menu and kept a time table. Common physical activity was allowed during the trial. The study lasted $16 \mathrm{~h}$, from 5:30 a.m. to 9:30 p.m.

Before the first blood sampling the subjects were given an intravenous cannula (in the cubital or forearm vein). The first blood withdrawal was at 6 a.m. (30 min after awaking) after overnight fasting. The next withdrawals were always one and two hours after eating according to the following schedule $(7: 15,8: 15,10: 15$, 11:15 a.m.; 1:15, 2:15, 4:15, 5:15, 7:15, 8:15 p.m.). The last blood withdrawal was $30 \mathrm{~min}$ before sleep, at 9:30 p.m. Breakfast ( 2 slice of bread, ham, cheese and tea) was served at 6:15, a snack (yogurt) at 9:15, lunch (beef broth soup, turkey, potato dumplings, and sauerkraut) at 12:15, an afternoon snack (apple) at 15:15, and dinner (tomato, 2 slices of bread, ham and cheese) at $18: 15$.

\section{Analytical methods}

Each sample was collected into a cooled plastic tube containing $100 \mu \mathrm{l}$ of $5 \% \mathrm{EDTA}$. The plasma was obtained after centrifugation for $5 \mathrm{~min}$ at $2000 \mathrm{rpm}$ at $4{ }^{\circ} \mathrm{C}$. Plasma was separated and frozen within half an hour of being drawn from the subject, and was stored at $-20{ }^{\circ} \mathrm{C}$ until analyzed.

C-peptide was measured in serum using ECLIA (electrochemiluminiscence immunoassay, Modular E
170 analyser, Roche). The measuring range of the kit (defined by the lower detection limit and the maximum of the master curve) was $0.003-13.3 \mathrm{nmol} / 1$ or 0.01 $40.0 \mathrm{ng} / \mathrm{ml}$ for plasma. Intra- and inter-assay coefficients of variation were $1.5 \%$ and $2.3 \%$, respectively.

Blood glucose was measured using the enzymatic reference method with hexokinase (Cobas Integra 400 plus analyser, Roche). The measuring range of the kit was $0.12-40 \mathrm{mmol} / \mathrm{l}(2.16-720 \mathrm{mg} / \mathrm{dl})$. Intraset and inter-set reproducibility were $1.7 \%$ and $2.6 \%$, respectively.

Total plasma ghrelin was determined by a commercially available RIA kit (Linco Research, Inc., St. Charles, Missouri, USA, detection range: 93-6000 $\mathrm{pg} / \mathrm{ml}$ ), and orexin A was also measured by a RIA kit (Phoenix Pharmaceuticals, Inc., detection range 10-1280 $\mathrm{pg} / \mathrm{ml})$.

Kits from Immunotech (France) were used to measure LH and FSH (IRMA kit). Cortisol was measured using RIA kit from Immunotech (France). Sex hormones bounding globulin (SHBG) were measured by the IRMA kit Immunotech (France).

\section{Steroids measured by GC/MS method}

The levels of unconjugated steroids were measured in cubital vein blood using the GC-MS method (Hill et al. 2010). In brief, free steroids were extracted from plasma by diethyl-ether; steroid conjugates were hydrolyzed and extracted. The resulting residues were derivatized by methoxyamine hydrochloride and analyzed by GC/MS as follows.

Steroids were purchased from Steraloids (Newport, RI, USA), Sylon B from Supelco (Bellefonte, PA, USA), methoxylamine hydrochloride from Sigma (St. Louis, MO, USA) and solvents from Merck (Darmstadt, Germany).

\section{Instruments}

Measurements of steroid levels were done on a GCMS-QP2010 Plus system by Shimadzu (Kyoto, Japan) consisting of a gas chromatograph equipped with automatic flow control, an AOC-20s autosampler, and a single quadrupole detector with an adjustable electron voltage of 10-195 V. A capillary column with a medium polarity RESTEK Rxi phase (diameter $0.25 \mathrm{~mm}$, length $15 \mathrm{~m}$, film thickness $0.1 \mu \mathrm{m}$ ) was used for analyses. Electron impact ionization with electron voltage fixed at $70 \mathrm{~V}$ and emission current set to $160 \mu \mathrm{A}$ was used. The 
temperatures of the injection port, ion source and interface were maintained at $220{ }^{\circ} \mathrm{C}, 300{ }^{\circ} \mathrm{C}$, and $310^{\circ} \mathrm{C}$, respectively. Analyses were carried out in the splitless mode with a constant linear velocity of the carrier gas $(\mathrm{He})$, which was maintained at $60 \mathrm{~cm} / \mathrm{s}$. The septum purge flow was set at $3 \mathrm{ml} / \mathrm{min}$. The samples were injected using the high pressure mode $(200 \mathrm{kPa})$, which was maintained for $1 \mathrm{~min}$. The detector voltage was set to $1.4 \mathrm{kV}$.

\section{Statistical data analysis}

The changes of steroid levels were evaluated using a repeated measures ANOVA model consisting of a Subject factor, explaining differences between subjects, and a Stage factor. Due to the non-Gaussian data distribution and non-constant variance, the original data were transformed by a power transformation to attain a symmetric distribution of the data and residuals as well as homoscedasticity (Meloun et al. 2000). The homogeneity of the transformed data was checked by residual analysis as described elsewhere (Meloun et al. 2002, 2004). Then, the significance of the values before and after food intake was evaluated by least significant differences multiple comparisons.

\section{Results}

The majority of the hormones showed significant differences of their levels during the day between hours and especially between subjects (Table 1). Non-significant differences between hours were only found for orexin, testosterone, and dihydrotestosterone (Table 1, H-F), and non-significant differences between subjects (Table 1, S-F) were only found for orexin, glucose and C-peptide.

A significant increase of glucose and C-peptide was observed 1 and $2 \mathrm{~h}$ following the main meals lunch and dinner $(\mathrm{P}=0.001$, Figs $1 \mathrm{~A}, \mathrm{~B}$; means and $95 \%$ confidence intervals are given in all figures). The most striking marker of food intake was the increase of C-peptide (Fig. 1B), which was significant after breakfast, lunch and dinner.

Ghrelin levels had a significant minimum after lunch and dinner (Fig. 1C), whereas the differences in orexin levels over the course of the day were insignificant (Fig. 1D, Table 1). LH and FSH (Fig. 2A,B) showed a continuous decrease during the day, with no significant additional decrease after main meals. Androgen levels (testosterone Fig. 3A, dihydrotestosterone Fig. 3B) did not show significant relationships to food intake, with the exception of an increase in dihydrotestosterone $2 \mathrm{~h}$ after lunch. Dehydroepiandrosterone (Fig. 3C) significantly decreased 1 and $2 \mathrm{~h}$ after lunch and breakfast respectively. SHBG (Fig. 5C) showed a small decrease after meals.

Table 1. Statistical characteristic of diurnal profiles of steroid hormones and other hormones/substances using repeated measures ANOVA with the factors Hour and Subject.

\begin{tabular}{lcccc}
\hline \multirow{2}{*}{ Substance } & \multicolumn{2}{c}{ Hour } & \multicolumn{2}{c}{ Subject } \\
\cline { 2 - 5 } & H-F & $\mathbf{p}$ & S-F & $\mathbf{p}$ \\
\hline Ghrelin & 3 & 0.0059 & 30.2 & $<0.0001$ \\
Orexin & 0.6 & 0.7789 & 2.3 & 0.0769 \\
Glucose & 4 & 0.0006 & 1.9 & 0.1235 \\
C-Peptide & 12.4 & $<0.0001$ & 2.7 & 0.0463 \\
LH & 3.1 & 0.0037 & 25.1 & $<0.0001$ \\
FSH & 3.9 & 0.0007 & 19.1 & $<0.0001$ \\
SHBG & 3.2 & 0.003 & 17.9 & $<0.0001$ \\
Cortisol & 16 & $<0.0001$ & 8.5 & $<0.0001$ \\
Progesterone & 7.3 & $<0.0001$ & 25.6 & $<0.0001$ \\
Pregnenolone & 5.7 & $<0.0001$ & 22.7 & $<0.0001$ \\
Testosterone & 1.7 & 0.1236 & 47.8 & $<0.0001$ \\
DHT & 1.9 & 0.0669 & 15.3 & $<0.0001$ \\
DHEA & 11.42 & $<0.0001$ & 12.4 & $<0.0001$ \\
Estrone & 10 & $<0.0001$ & 122.1 & $<0.0001$ \\
Estradiol & 2.8 & 0.0101 & 41.5 & $<0.0001$ \\
\hline
\end{tabular}

$\mathrm{H}-\mathrm{F}$ - Hour factor showing the differences between time periods, $\mathrm{S}-\mathrm{F}$ - Subject factor showing the differences between individual subjects, $\mathrm{p}$ - significance.

All three C21 steroids (cortisol Fig. 4A, progesterone Fig. 4B and pregnenolone Fig. 4C) showed a significant decrease one hour after awakening and then a continuous decrease during the day. Cortisolemia decreased continuously throughout the day, and an additional significant decrease of cortisol related to food intake was only observed $2 \mathrm{~h}$ after lunch (Fig. 4A).

Estrogens (estrone Fig. 5A and estradiol Fig. 5B) did not show any striking trend in daily profiles; estradiol decreased significantly after lunch and dinner. 

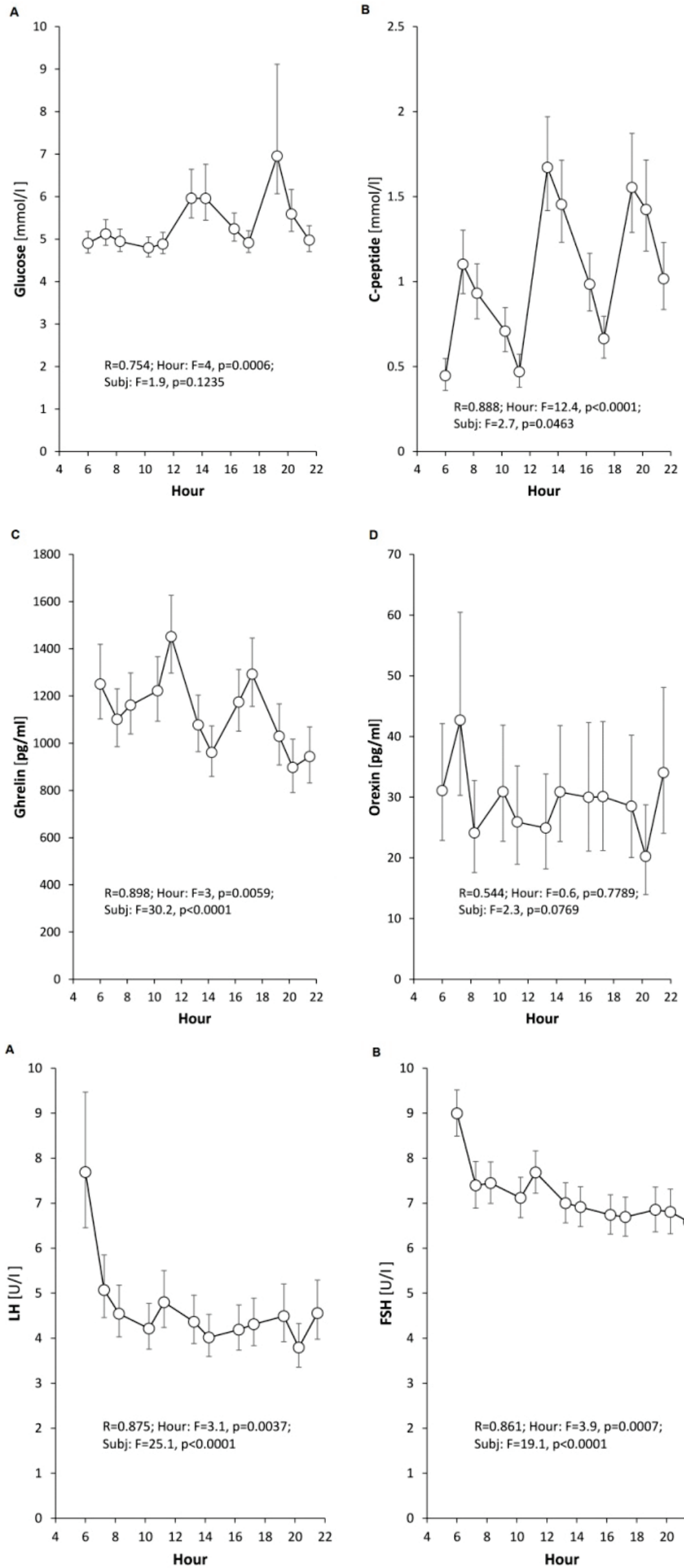

B
Fig. 1. Daily profiles of glucose (A), C-peptide (B), and the food-related hormones ghrelin (C) and orexin (D).

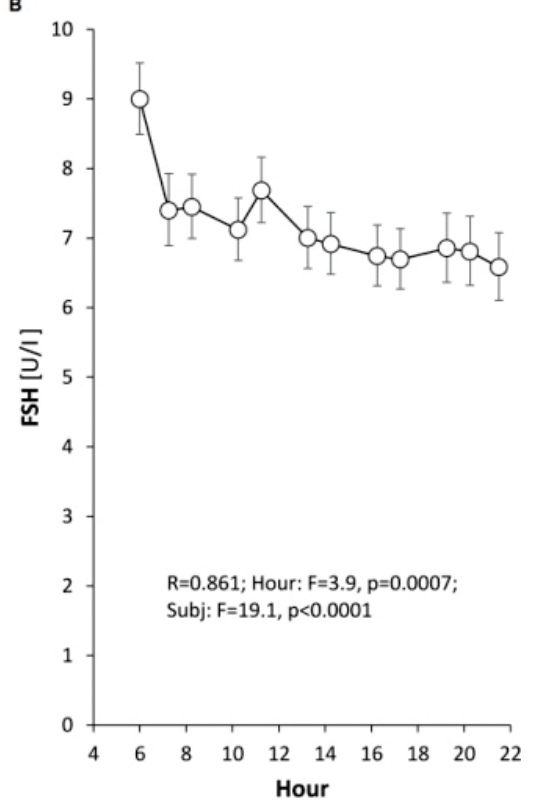

Fig. 2. Daily profiles of the gonadotropins $\mathrm{LH}$ (A) and FSH (B). 

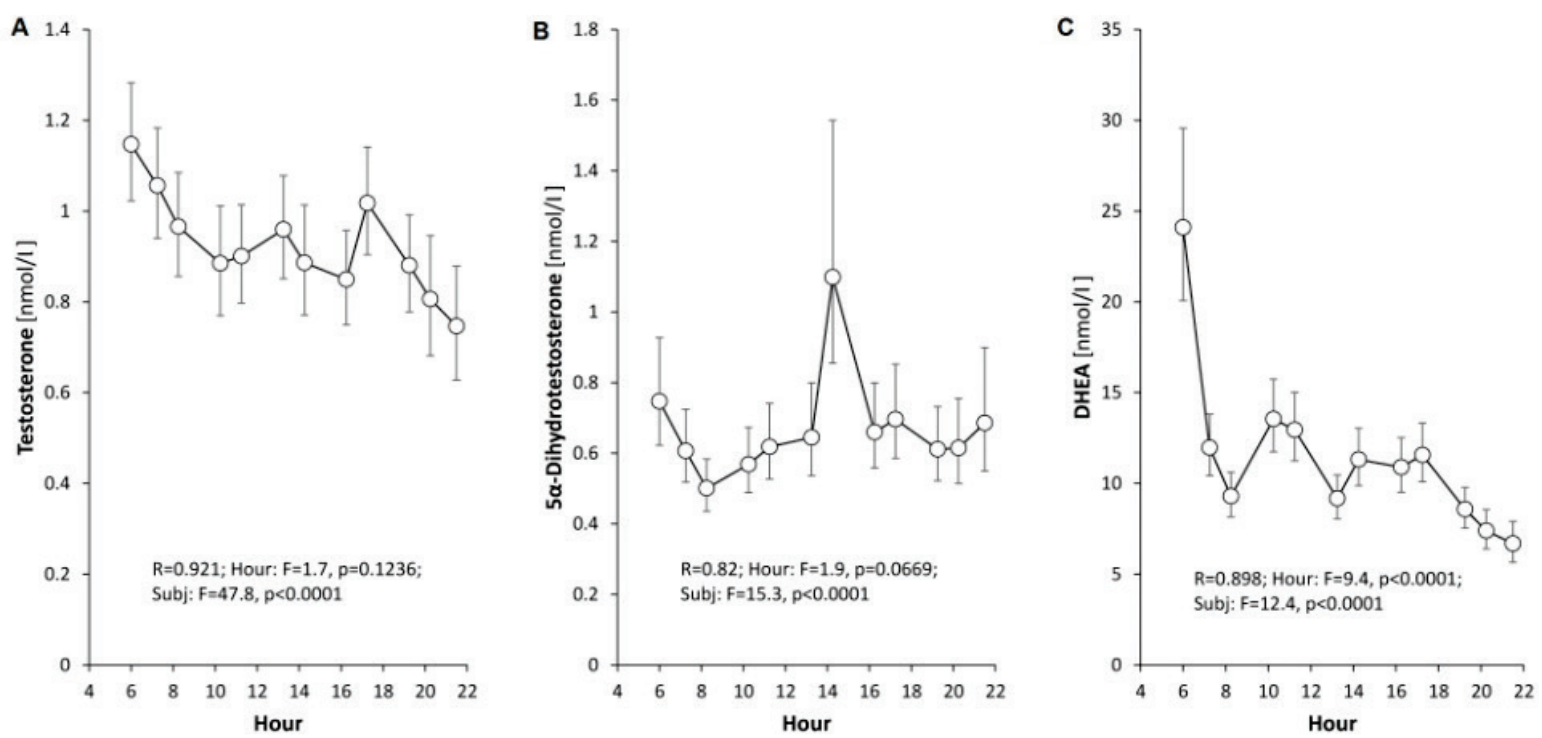

Fig. 3. Daily profiles of the androgens testosterone (A), dihydrotestosterone (B) and dehydroepiandrosterone (C).
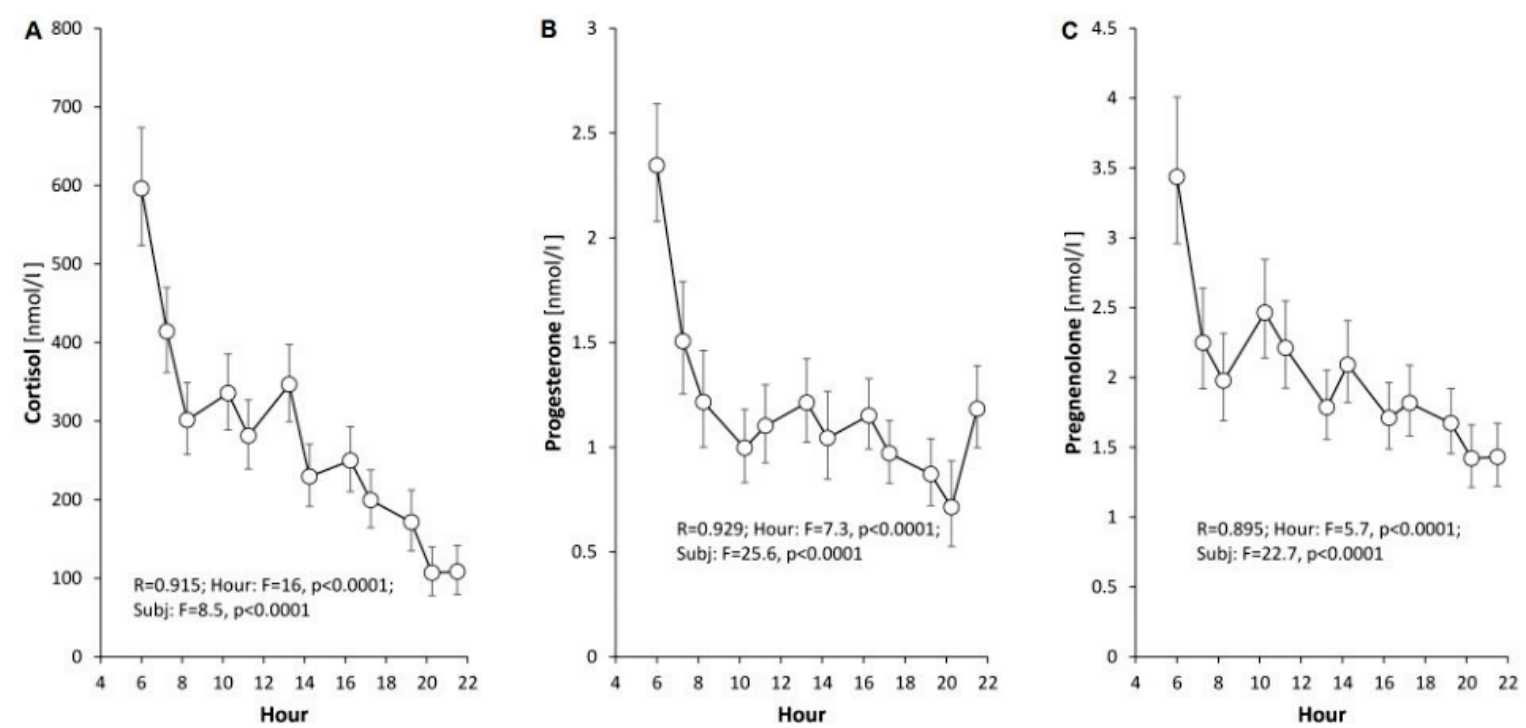

Fig. 4. Daily profiles of the $C 21$ steroids cortisol (A), progesterone (B) and pregnenolone (C).
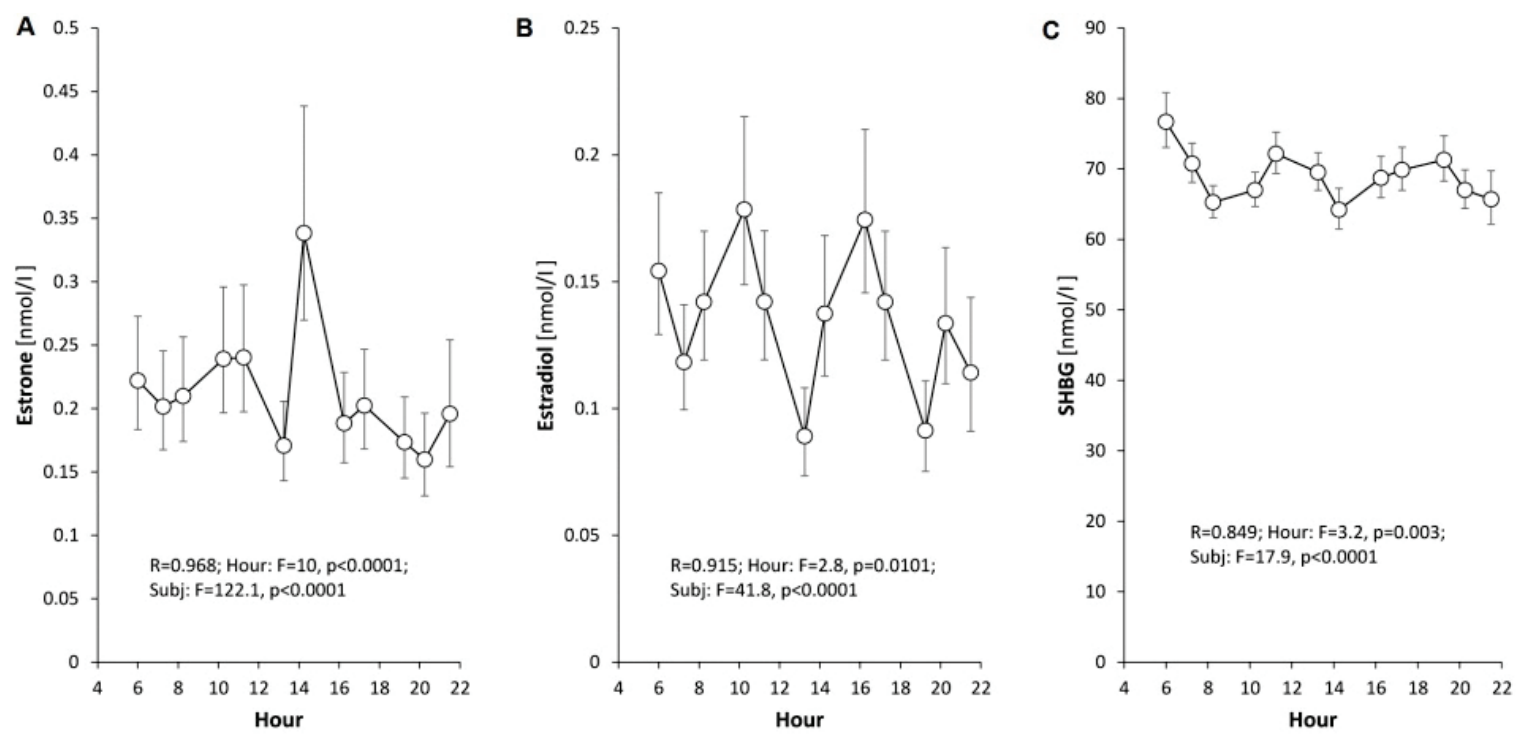

Fig. 5. Daily profiles of the estrogens estrone (A) and estradiol (B), and SHBG (C). 


\section{Discussion}

Whereas the daily profiles of the main steroid hormones are well known, minor differences in the course of their levels related to defined and standardized food intake have not been studied systematically. In contrast to plenty of papers dealing with the effects of steroid hormones on eating behavior and disorders, the effect of food intake - with the exception of the effect of diet on endocrine homeostasis - has not yet received much attention in the literature. In animals, meal timing is a powerful synchronizer; however, there are no definitive and conclusive data to prove that meal timing is also a true synchronizer in humans, although there have been some reports suggesting this (Sensi et al. 1993). Meal timing was found to be an important factor determining the leptin diurnal rhythm, but other factors must contribute since the leptin response to a standard meal taken in the evening was greater than to the same meal taken in the morning (Elimam and Marcus 2002). The effects of two different feeding regimes on the 24-h profiles of maternal and fetal plasma cortisol and adrenocorticotrophic hormone concentrations were studied in pregnant ewes (Simonetta et al. 1991). There were differences between the time of maximal glucose concentrations in maternal and fetal plasma in the oncedaily-fed ewes in contrast to the multi-fed group. A significant diurnal variation in the plasma concentrations of cortisol was also present in the oncedaily-fed ewes dependent on the stage of gravidity.

The timing of food intake is associated with body weight regulation, insulin sensitivity and glucose tolerance, but the mechanisms are unknown. Bandín et al. (2015) found that eating late is associated not only with decreased resting-energy expenditures, decreased fasting carbohydrate oxidation and decreased glucose tolerance, but also smoothed out the daily profile of free cortisol concentrations.

In our study the known nycthemeral rhythm of LH, FSH, cortisol, dehydroepiandrosterone, progesterone and pregnenolone and decrease of ghrelin after food intake were confirmed, but significant changes after meals were also newly observed for the levels of cortisol, dehydroepiandrosterone, estradiol and SHBG. These effects were seen only after the main meals lunch and dinner. After breakfast, these effects are probably masked by the huge changes in the nycthemeral rhythm, and after snacks (yogurt and apples) the effects of food intake were either too small or the time period between eating the meal and the blood withdrawal was too long. It follows that for analytical determination of above mentioned hormones not only the time of blood withdrawals but also food consumption has an important influence upon the resulting values.

\section{Conflict of Interest}

There is no conflict of interest.

\section{Acknowledgements}

The study was supported by grant no. NT-12340-5 of the Internal Grant Agency of the Ministry of Health, Czech Republic.

\section{References}

BANDÍN C, SCHEER FA, LUQUE AJ, AVILA-GANDÍA V, ZAMORA S, MADRID JA, GÓMEZ-ABELLÁN P, GARAULET M: Meal timing affects glucose tolerance, substrate oxidation and circadian-related variables: A randomized, crossover trial. Int J Obes (Lond) 39: 828-833, 2015.

CHRISTIAN CA, MOENTER SM: The neurobiology of preovulatory and estradiol-induced gonadotropin-releasing hormone surges. Endocr Rev 31: 544-577, 2010.

CHUNG S, SON GH, KIM K: Circadian rhythm of adrenal glucocorticoid: its regulation and clinical implications. Biochim Biophys Acta 1812: 581-591, 2011.

DICKMEIS T: Glucocorticoids and the circadian clock. $J$ Endocrinol 200: 3-22, 2009.

ELIMAM A, MARCUS C: Meal timing, fasting and glucocorticoids interplay in serum leptin concentrations and diurnal profile. Eur J Endocrinol 147: 181-188, 2002.

FORSLING ML: Diurnal rhythms in neurohypophysial function. Exp Physiol 85 (Spec No): 179S-186S, 2000.

HILL M, PAŘÍZEK A, KANCHEVA R, DUŠKOVÁ M, VELÍKOVÁ M, KŘÍŽ L, KLÍMKOVÁ M, PAŠKOVÁ A, ŽIŽKA Z, MATUCHA P, MELOUN M, STÁRKA L: Steroid metabolome in plasma from the umbilical artery, umbilical vein, maternal cubital vein and in amniotic fluid in normal and preterm labor. $J$ Steroid Biochem Mol Biol 121: 594-610, 2010. 
KALSBEEK A, FLIERS E: Daily regulation of hormone profiles. Handb Exp Pharmacol 217: 185-226, 2013.

KARATSOREOS IN, WANG A, SASANIAN J, SILVER R: A role for androgens in regulating circadian behavior and the suprachiasmatic nucleus. Endocrinology 148: 5487-5495, 2007.

KONTUREK PC, BRZOZOWSKI T, KONTUREK SJ: Gut clock: implication of circadian rhythms in the gastrointestinal tract. J Physiol Pharmacol 62: 139-150, 2011.

MELOUN M, HILL M, MILITKÝ J, KUPKA AK: Transformation in the PC-aided biochemical data analysis. Clin Chem Lab Med 38: 553-559, 2000.

MELOUN M, MILITKÝ J, HILL M, BRERETON RG: Crucial problems in regression modelling and their solutions. Analyst 127: 433-450, 2002.

MELOUN M, HILL M, MILITKÝ J, VRBÍKOVÁ J, STANICKÁ S, ŠKRHA J: New methodology of influential point detection in regression model building for the prediction of metabolic clearance rate of glucose. Clin Chem Lab Med 42: 311-322, 2004.

PATTON DF, MISTLBERGER RE: Circadian adaptations to meal timing: neuroendocrine mechanisms. Front Neurosci 7: 185, 2013.

SENSI S, PACE PALITTI V, GUAGNANO MT: Chronobiology in endocrinology. Ann Inst Super Sanita 29: 613-631, 1993.

SIMONETTA G, WALKER DW, MCMILLEN IC: Effect of feeding on the diurnal rhythm of plasma cortisol and adrenocorticotrophic hormone concentrations in the pregnant ewe and sheep fetus. Exp Physiol 76: 219-229, 1991.

SON GH, CHUNG S, KIM K: The adrenal peripheral clock: glucocorticoid and the circadian timing system. Front Neuroendocrinol 32: 451-465, 2011.

STÁRKA L, RÁCZ B, ŠRÁMKOVÁ M, HILL M, DUŠKOVÁ M: Daily profiles of dehydroepiandrosterone and its hydroxylated metabolites with respect to food intake. Prague Med Rep 116: 40-48, 2015.

TRIVEDI AK, KUMAR V: Melatonin: an internal signal for daily and seasonal timing. Indian J Exp Biol 52: 425-437, 2014.

VANĚČEK J: Cellular mechanisms of melatonin action. Physiol Res 78: 687-721, 1998. 